\title{
RNAi-mediated inhibition of apoptosis fails to prevent cationic nanoparticle-induced cell death in cultured cells
}

\begin{abstract}
Background: Nanoparticles are increasingly being considered as a novel and potent tool for drug delivery, and, therefore, concerns regarding the safety of their use in humans are pertinent. It has been shown that nanoparticles displaying unsaturated amines at their surface are toxic to cells, but the molecular and cellular mechanisms elicited in this response have yet to be described. Aims: In this work we identify key proteins involved in the cytotoxicity of amine-modified polystyrene nanoparticles. We also demonstrate the suitability of RNAi to provide a molecular description of how nanoparticles and cells interact. Materials \& methods: We have used a focused RNAi strategy in $1321 \mathrm{~N} 1$ cells to identify key proteins involved in the cytotoxicity induced by amine-modified polystyrene nanoparticles. Results: We show that the apoptosome is central to the observed mechanism of toxicity and that, although the proapoptotic proteins BAX, BAK, BID, BIM and PUMA are critical modulators of the process, their cellular depletion is insufficient to protect cells from nanoparticleinduced cell death. Conclusion: We conclude that the apoptosome, together with proapoptotic proteins of the $\mathrm{BCl}-2$ family of proteins, is central to amine-modified polystyrene nanoparticle-induced cell death. We further demonstrate that RNAi is a powerful and suitable tool to study the effects of nanoparticles on cellular processes, in particular apoptosis.
\end{abstract}

Original submitted 18 March 2013; Revised submitted 22 July 2013

Keywords: apoptosis $\bullet$ caspase $\bullet$ cationic nanoparticle $\bullet$ nanotoxicity $\bullet$ RNAi

Despite being a relatively new field, nanotechnology is already being applied in a clinical context, in areas such as cancer therapy, vascular interventions, theranostics and diagnostic imaging [1-4]. However, despite the use of nanoparticles in several of these types of applications, there remain concerns about their use [5]. As a prerequisite to their wider use, it is essential that we characterize the breadth of interactions that nanoparticles have with their surroundings at both the cellular and molecular level. In this regard, understanding the signals that are elicited by cells on exposure to nanoparticles is an essential part of their characterization prior to clinical application.

Cationic nanoparticulates, including liposomes and polyplexes, have been shown previously to be toxic to cells [6-8]. We and others have shown that engineered cationic nanoparticles can interact with mammalian cells and induce apoptosis [9,10]. However, indepth studies of the molecular and cellular mechanisms underlying these events have been lacking. Using amine-modified polystyrene ( $\left.\mathrm{NH}_{2}-\mathrm{PS}\right)$ nanoparticles of $50 \mathrm{~nm}$ in diameter as a model for cationic nanoparticles we have previously reported that they are capable of rapidly inducing cell death in a $1321 \mathrm{~N} 1$ human astrocytoma cell line [9]. In these initial studies, we used a chemiluminescence-based assay to suggest a role for caspases (CASPs) in the toxicity mechanism induced by these $\mathrm{NH}_{2}$-PS nanoparticles [9]. However, the biochemical tools to
Mariana G Bexiga ${ }^{1,2,3}$, Ciara Kelly ${ }^{1}$, Kenneth A Dawson ${ }^{3}$ \& Jeremy C Simpson*,1 ${ }^{1}$ School of Biology \& Environmental Science \& Conway Institute of Biomolecular \& Biomedical Research, University College Dublin, Dublin 4, Ireland

${ }^{2}$ Centre for Neuroscience \& Cell Biology, University of Coimbra, Portugal ${ }^{3}$ Centre for BioNano Interactions, School of Chemistry \& Chemical Biology, University College Dublin, Dublin 4, Ireland

*Author for correspondence: Tel.: +35317162345 Fax: +35317161153 jeremy.simpson@ucd.ie 
further elucidate the role of these CASPs are somewhat limited, and there is evidence that the CASP substrates and inhibitors currently available show cross-specificity, in particular towards CASP-3 [11]. We, therefore, decided to adopt a more specific strategy, namely the use of RNAi, to delineate the molecular events associated with nanoparticle-induced CASP activation and cell death.

The complete sequencing of the human genome, its detailed level of annotation [12], and the discovery of RNAi and its ability to specifically and rapidly silence individual genes [13-15] have been key events in the study of cellular function. Large-scale RNAi studies have been successfully employed to dissect a variety of cellular pathways, including cell division [16], protein secretion [17] and apoptosis [18]. RNAi is a highly conserved innate mechanism of gene-specific gene silencing that works via the targeted destruction of individual mRNA molecules by homologous double-stranded siRNAs [13,15]. These reagents can be generated by chemical synthesis in vitro, or in vivo by transcription through vector-based expression systems, and have proven to be very useful tools in study of gene loss of function in mammalian cells [15].

Here, we use a highly focused RNAi screen to identify key proteins involved in the cytotoxicity induced by $\mathrm{NH}_{2}-\mathrm{PS}$ nanoparticles in $1321 \mathrm{~N} 1$ cells. We use this biological system because it has been reported that some nanoparticles present in pollution are positively charged [19]. Similar particles not only affect the respiratory tract, but may also cross to the brain causing inflammation, deposition of amyloid- $\beta$ and other pathological events [20], which are, in turn, characteristic of neurological diseases such as Alzheimer's and Parkinson's disease [21]. In addition, positively charged particles have been used in diagnostic and therapeutic applications [3]. We provide evidence that siRNA can be used to study the mechanisms of interaction of nanoparticles with cells. We also confirm that the apoptosome is central to the observed mechanism of toxicity and show that proapoptotic proteins BAX, BAK, BID, BIM and PUMA are critical modulators of the process. Their depletion is, however, insufficient to rescue cells treated with $\mathrm{NH}_{2}$-PS nanoparticles from cell death.

\section{Materials \& methods}

Cell culture \& nanoparticles

Human brain astrocytoma $1321 \mathrm{~N} 1$ cells were cultured at $37^{\circ} \mathrm{C}$ in DMEM containing $4.5 \mathrm{~g} / \mathrm{l}$ of D-glucose and $1 \mathrm{mM}$ of sodium pyruvate supplemented with $10 \%$ fetal bovine serum (Life Technologies, CA, USA). Cells were used from passages 1-10, after which they were discarded. $\mathrm{NH}_{2}$-PS fluorescent polystyrene nano- particles with a diameter of $50 \mathrm{~nm}$ (Sigma Aldrich, MO, USA) were used without further modification. Nanoparticles of the same batch were used throughout this work. This particular batch of nanoparticles has been previously characterized by electron microscopy, dynamic light scattering and $\zeta$-potential analysis [9]. These analyses revealed that these nanoparticles were well dispersed in phosphate-buffered saline, showing a polydispersity index of 0.11 . Their diameter was measured by both electron microscopy and dynamic light scattering and was found to be $46.7 \pm 8.1 \mathrm{~nm}$ and $55.1 \mathrm{~nm}$ according to these two methods, respectively. These measurements were, therefore, in good agreement with the supplier's annotation of them being $50 \mathrm{~nm}$ in diameter. $\zeta$-potential measurements indicated that these nanoparticles displayed a surface charge of $+25.8 \mathrm{mV}$.

\section{siRNA transfection}

Cells were transfected with 30 pmol of Silencer ${ }^{\circledR}$ Select siRNA (Supplementary Table 1; see online at www. futuremedicine.com/doi/suppl/10.2217/NNM.13.151; Life Technologies) using Oligofectamine ${ }^{\mathrm{TM}}$ (Life Technologies) according to the manufacturer's instructions. Cells were transfected with siRNAs for $48 \mathrm{~h}$ in all experiments.

\section{Total RNA extraction, cDNA synthesis}

\& real-time quantitative PCR

Total RNA from $1321 \mathrm{~N} 1$ cells, treated as described above, was purified using the Invisorb ${ }^{\circledR}$ spin cell RNA mini kit (STRATEC Molecular, Berlin, Germany) as recommended by the supplier. RNA concentrations were determined using a NanoDrop3000 (Thermo Scientific, MA, USA). cDNA synthesis was performed with 500 ng of total RNA using the High Capacity cDNA Reverse Transcription kit (Life Technologies) according to the supplier's instructions. Real-time quantitative PCR (qPCR) was performed using Power $\mathrm{SYBR}^{\circledR}$ green PCR MasterMix (Life Technologies) in a 7500 FAST real-time PCR system (Life Technologies). A twentieth of the cDNA reaction was used as a template for the reaction and $200 \mathrm{nM}$ of each primer (Supplementary Table 2) was used. All samples were run in quadruplicate. Results were obtained using the $-\Delta \mathrm{Ct}$ method, with mRNA levels from specific siRNA-treated cells being normalized to those found in cells treated with nonsilencing (NEG) siRNAs.

\section{Preparation of whole-cell extracts \& western blotting}

Following incubation with nanoparticles, cells were lysed with RIPA buffer (Thermo Scientific) supplemented with Complete ${ }^{\mathrm{TM}}$ protease inhibitor cocktail 
tablets (Roche, Basel, Switzerland). Proteins from each sample were quantified using the BCA protein assay kit (Thermo Scientific) and were then subjected to SDSPAGE gel electrophoresis. The proteins were transferred to polyvinylidene fluoride membrane (Perkin Elmer, MA, USA) and detected using primary antibodies (anti-BID, -BOK, -CASP-7, -CASP-9, -GAPDH, -PARP-1 and -PUMA purchased from Cell Signaling Technologies; MA, USA; anti-BAK, -BIM, -CASP-3 and -CASP-8 from BD Pharmingen; CA, USA; antiAPAF1 and -BAX from BD Transduction Laboratories; CA, USA; and anti-CASP10 and-GAPDH from Abcam, Cambridge, UK), followed by incubation with secondary antibodies coupled to either HRP (Abcam) or ALKP (Sigma Aldrich). Proteins were detected with Attophos ${ }^{\circledR}$ fluorescent reagent (Roche) or Pierce ECL western blotting substrate (Thermo Scientific). To quantify the amount of cleaved product in PARP-1 western blots, Image J 1.42q (NIH, MD, USA) software was used. Briefly, for each lane, the background, uncleaved and cleaved bands were quantified using the same rectangular selection for all. Results were expressed as the percentage of the cleaved protein over the total amount of protein (cleaved + uncleaved protein).

\section{Measurement of CASP-3 and -7 activities $\&$ cellular ATP content}

Measurement of CASP-3 and CASP-7 activities was carried out using the Caspase-Glo ${ }^{\circledR}$ 3/7 assay (Promega, WI, USA) and cellular ATP content was determined using the CellTiter-Glo ${ }^{\circledR}$ assay (Promega), according to the manufacturer's instructions. Briefly, cells were plated in a 96-well plate and transfected for $48 \mathrm{~h}$ with siRNAs. The cells were then treated with a $50-\mu \mathrm{g} / \mathrm{ml}$ nanoparticle dispersion for $24 \mathrm{~h}$ at $37^{\circ} \mathrm{C}$. After incubation, an equal volume of the assay reagent was added to the cells and incubation was continued for a further $1 \mathrm{~h}$ at room temperature. Luminescence was measured using a Wallac VICTOR2 ${ }^{\mathrm{TM}}, 1420$ Multilabel Counter (Perkin Elmer). Results were normalized either against the untreated or negative siRNA controls.

\section{Analysis of mitochondrial morphology}

$1321 \mathrm{~N} 1$ cells $\left(1 \times 10^{5}\right.$ cells $)$ were plated on glass coverslips previously coated with rat tail collagen type I (Sigma Aldrich). They were then transfected for $48 \mathrm{~h}$ with siRNA as described above and incubated for a further $6 \mathrm{~h}$ with $50 \mu \mathrm{g} / \mathrm{ml}$ of $\mathrm{NH}_{2}$-PS nanoparticles. Following methanol fixation, cells were stained with mouse anti-HSP60 antibodies (BD Biosciences) followed by secondary staining with antimouse antibodies conjugated to Alexa-568 (Molecular Probes,
OR, USA) and nuclei were stained with Hoechst 33342 (Sigma Aldrich). Confocal images were acquired using an Olympus FluoView FV1000 confocal microscope with a $60 \times 1.35$ NA Olympus UPlanSAPO oil immersion objective (Olympus Corporation, Tokyo, Japan). Cell outlines and mitochondria were segmented based on the signal in the Alexa-568 channel, followed by mitochondrial number and morphology quantification using Columbus Image Analysis software (Perkin Elmer). Multiple parameters were measured per cell to distinguish phenotypes. Briefly, cell outlines and mitochondria were segmented based on the signal in the Alexa-568 channel, followed by the quantification of cell area and mitochondrial number and morphology. Mitochondrial counts were adjusted by cell area and normalized to untreated groups. Roundness measurements were also normalized to untreated groups with higher values representing increasing roundness. A minimum of 24 cells per condition were used for analysis, and between 24 and 47 individual mitochondrial elements (according to their segmentation) were quantified per cell, meaning that a minimum of 600 mitochondrial elements per condition were measured.

\section{Statistical analysis}

Analysis of variance (one-way ANOVA) was performed to compare the experimental conditions. Post hoc analysis was carried out using Tukey's test. All statistical analyses were performed with either PASW Statistics v.18 software (IBM Corporation, NY, USA) or Prism v4 (GraphPad Software, Inc., CA, USA).

\section{Results}

Establishment of a RNAi strategy to study cationic nanoparticle-induced apoptosis

Initially, an RNAi strategy was optimized for the use in our experimental system. The first targets chosen were those involved in the initiation (CASP-8, -9 and -10 and APAF1) and execution (CASP-3 and -7) of the apoptotic cascades. Real-time qPCR analysis of mRNA levels from the cell populations treated with the panel of siRNAs (using two independent sequences to target each gene) revealed that, with the exception of siRNAs targeting CASP-10, all the siRNAs depleted their target mRNAs by $70 \%$ or greater over the $48 \mathrm{~h}$ of the experiment (Figure 1A). Levels of CASP-10 transcripts were only reduced to $50 \%$ of those seen in control cells and so we tested a third siRNA targeting this gene. This sequence was successfully able to deplete its target mRNA to approximately $30 \%$ of control levels (Figure 1A) and so this reagent was used in subsequent studies testing CASP10 involvement in nanoparticle-induced cell death. 


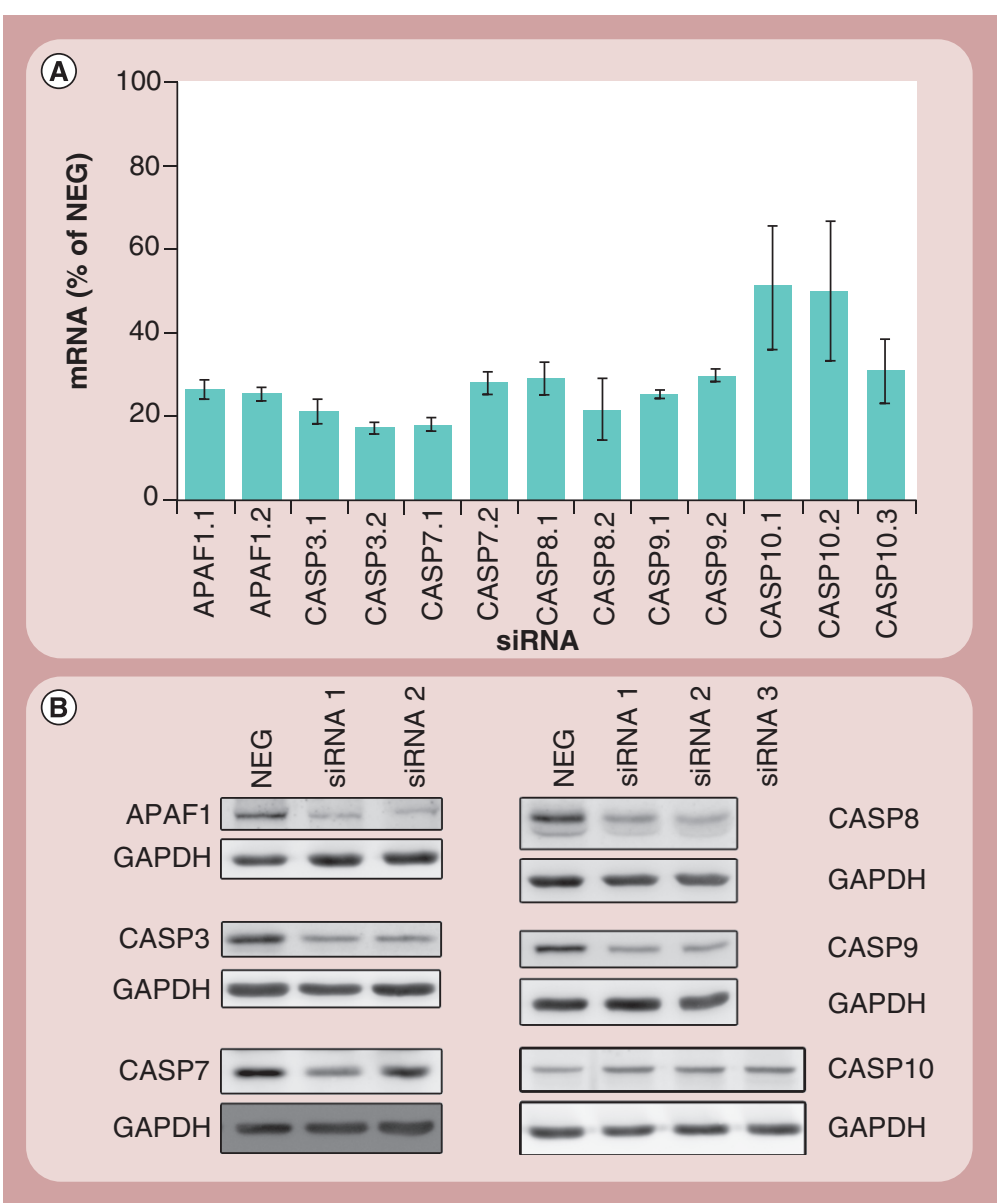

Figure 1. Depletion of apoptotic pathway components using RNAi.

(A) Validation of the efficiency of the siRNA knockdown at the mRNA level by real-time qPCR as described in the 'Materials \& methods' section. Bars indicate relative mRNA levels after $48 \mathrm{~h}$ in cells treated with various siRNAs compared with cells treated with NEG siRNAs. Results shown represent the mean and standard deviations of four technical replicates and are representative of three independent experiments. All data points were significantly different $(p<0.001)$ from cells treated with NEG siRNAs.

(B) Western blotting showing the efficiency of the siRNA knockdown at the protein level $48 \mathrm{~h}$ post-transfection. GAPDH is used as a loading control. CASP: Caspase; qPCR: Quantitative PCR.

Although qPCR provides a useful indication of efficacy of siRNA-mediated gene knockdown, many cellular proteins are known to have long half-lives and, therefore, a more accurate assessment of siRNA activity is to measure protein levels in the cells following siRNA treatment. To this end, we prepared protein extracts from the cells incubated with the various siRNAs, and then assessed protein levels by SDS-PAGE and western blotting. For the majority of the extracts tested, we observed a strong correlation between the extent of protein depletion and the reduction of mRNA levels as recorded in the qPCR experiments (Figure 1B). The depletion of CASP-10 protein was the least effective, in agreement with the qPCR data, despite three independent siRNAs being tested.
We next applied our siRNA panel in the context of a luminescence-based assay that assesses the activation of CASP-3 and -7, and, therefore, apoptosis. To test that this assay was compatible with siRNA-treatment of cells, we first performed a pilot experiment in either untreated cells or cells incubated with a NEG siRNA, followed by treatment with nanoparticles. Following the $48 \mathrm{~h}$ of siRNA treatment, but prior to nanoparticle addition, visual inspection of the cells revealed no apparent toxic effects of the negative control siRNA (not shown). We therefore incubated the cells with $50 \mu \mathrm{g} / \mathrm{ml}$ of $\mathrm{NH}_{2}$-PS nanoparticles for a period of $24 \mathrm{~h}$, and then measured the levels of CASP- 3 and -7 activation. This concentration was previously shown to induce apoptosis in $1321 \mathrm{~N} 1$ cells treated for $24 \mathrm{~h}$ with $\mathrm{NH}_{2}$-PS nanoparticles (Supplementary Figure 1) [9]. Similar to what we have described previously [9], the addition of this class of nanoparticles to the cells resulted in a significant CASP activation response. However, importantly, these experiments also revealed no discernible difference in the level of activation of these CASPs between the cells not treated with siRNAs and those treated with NEG siRNAs (Figure 2A).

\section{The apoptosome is central to the apoptotic mechanism}

To analyze phenotypic effects in the context of CASP depletion, these assays were initially repeated in cells treated with siRNAs targeting CASPs common to both the intrinsic and extrinsic apoptosis pathways, namely CASP-3 and -7. As expected, depletion of either of these targets in cells treated with nanoparticles resulted in a significant reduction in their activation as reported by the luminescence-based assay (Figure 2B). Of the four individual siRNAs we tested, the two siRNAs targeting CASP-3 produced a stronger effect, with just one of the siRNAs (CASP-7.2) showing no effect. Unsurprisingly, this siRNA was the sequence previously shown to be the poorest in downregulating its target (Figure 1A \& B). We next applied the same strategy to assess the relative roles of the extrinsic and intrinsic apoptosis pathways in nanoparticle-induced cell death. RNAi-mediated downregulation of CASP-8 and -10 did not significantly reduce activation of our reporter CASPs, although consideration should be given to the fact that the efficacy of siRNAs targeting CASP-10 was only validated at the mRNA level (Figure 1A \& B). Nevertheless, as CASP-8 and -10 function together, and CASP- 8 was efficiently depleted by our reagents, these results suggest that the extrinsic pathway does not contribute to apoptosis (Figure 2C). By contrast, when these experiments were repeated, but this time depleting proteins specific to the intrinsic apoptosis pathway (CASP-9 and APAF1), 
we observed a strong and significant effect on CASP-3 and -7 activation (Figure 2D).

In order to strengthen these observations, we repeated our experiments, combining pairs of siRNAs acting on the same apoptosis pathway. Combined depletion of CASP-8 and -10 (extrinsic pathway) showed no reduction in reporter CASP activation, whereas depletion of CASP-9 and APAF1 (intrinsic pathway), or indeed the reporter CASPs (CASP-3 and -7), resulted in a marked loss of apoptotic activity by almost $70 \%$ in the $1321 \mathrm{~N} 1$ cells treated with nanoparticles (Figure 2E). To further evaluate the sensitivity of this strategy, we treated cells for $6 \mathrm{~h}$ with $1 \mu \mathrm{M}$ of staurosporine, a well-known inducer of apoptosis that leads to activation of CASP-3 [22]. These experiments revealed that even under these potent apoptotic-inducing conditions, siRNA combinations targeting CASP-3 and -7, -9 and APAF1, for example, were still able to abrogate apoptosis by a significant degree (Supplementary Figure 2).

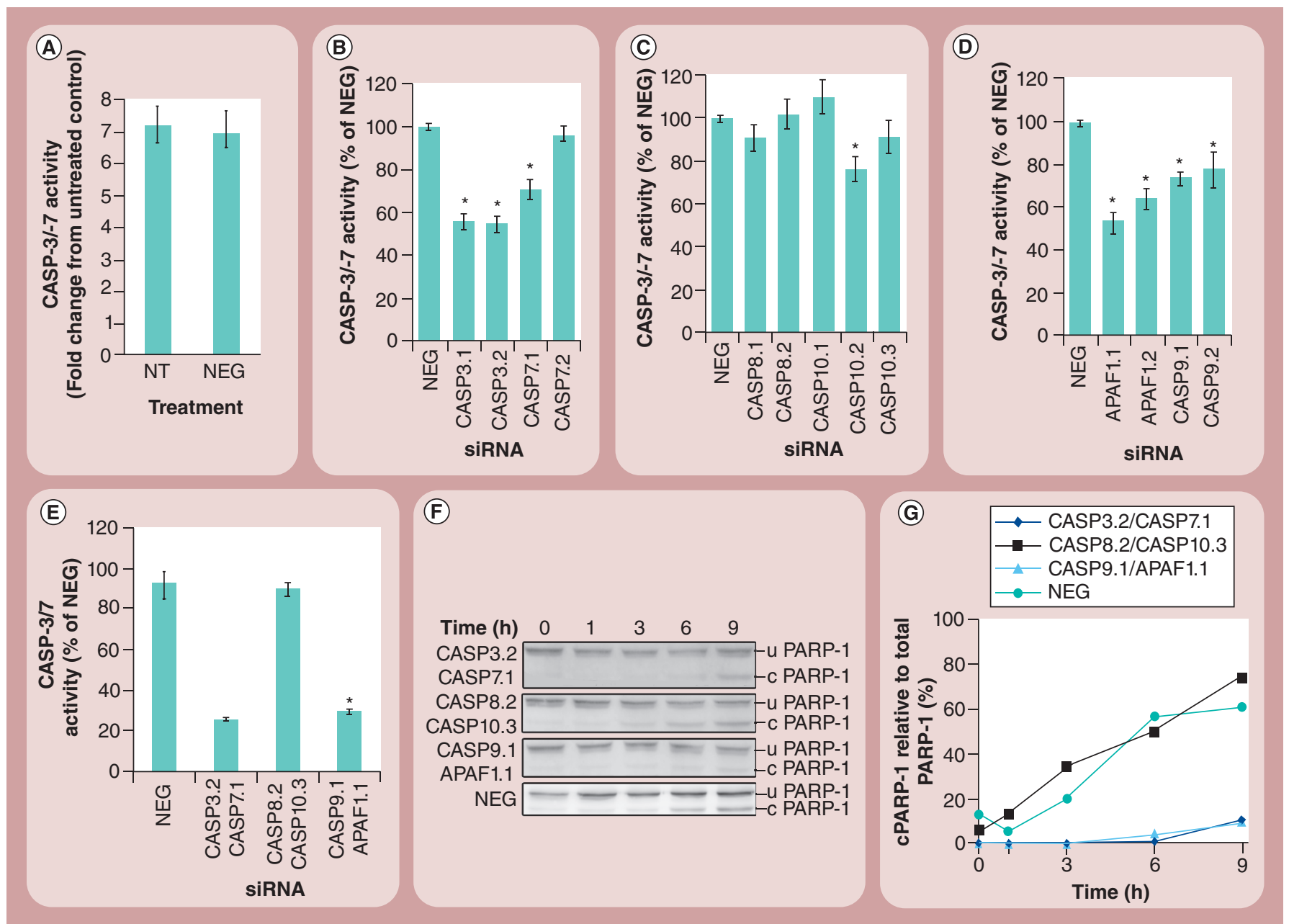

Figure 2. Apoptosome formation is central to the apoptotic pathway induced by amine-modified polystyrene nanoparticles.

(A) CASP-3 and -7 activities of $1321 \mathrm{~N} 1$ cells nontransfected or transfected with NEG siRNA for $48 \mathrm{~h}$ followed by incubation with $50 \mathrm{\mu g} / \mathrm{ml}$ of amine-modified polystyrene ( $\mathrm{NH}_{2}-\mathrm{PS}$ ) nanoparticles for $24 \mathrm{~h}$. (B) CASP-3 and -7 activities of $1321 \mathrm{~N} 1$ cells treated for $48 \mathrm{~h}$ with siRNAs targeting CASP-3 and-7 followed by $24 \mathrm{~h}$ of incubation with $\mathrm{NH}_{2}$-PS nanoparticles are shown as a percentage of the activity of cells treated with NEG siRNAs. (C) CASP-3 and -7 activities of cells treated for $48 \mathrm{~h}$ with siRNAs targeting CASP-8 and -10 followed by $24 \mathrm{~h}$ of incubation with $\mathrm{NH}_{2}$-PS nanoparticles are shown as a percentage of the activity of cells treated with NEG siRNAs. (D) CASP-3 and -7 activities in cells treated for $48 \mathrm{~h}$ with siRNAs targeting APAF1 and CASP9 followed by $24 \mathrm{~h}$ of incubation with $\mathrm{NH}_{2}$-PS nanoparticles are shown as a percentage of the activity of cells treated with NEG siRNAs. (E) CASP-3 and -7 activities in cells transfected for $48 \mathrm{~h}$ with siRNAs targeting two molecules in the same apoptotic pathway followed by $24 \mathrm{~h}$ of incubation with $\mathrm{NH}_{2}$-PS nanoparticles are shown as a percentage of the activity of cells treated with NEG siRNAs. (F) $1321 \mathrm{~N} 1$ cells were treated as for (D) and $30 \mu \mathrm{g}$ of protein was then subjected to western blotting to detect PARP-1 cleavage. (G) Quantification of the percentage of cleaved PARP-1 over the total PARP-1 protein was carried out by densitometry from the western blots presented in (F). Results represent the mean and standard error of the mean from three independent experiments, each with three replicates.

* $p<0.001$.

C PARP-1: Cleaved PARP-1; CASP: Caspase; NT: Nontransfected; u PARP-1: Uncleaved PARP-1. 

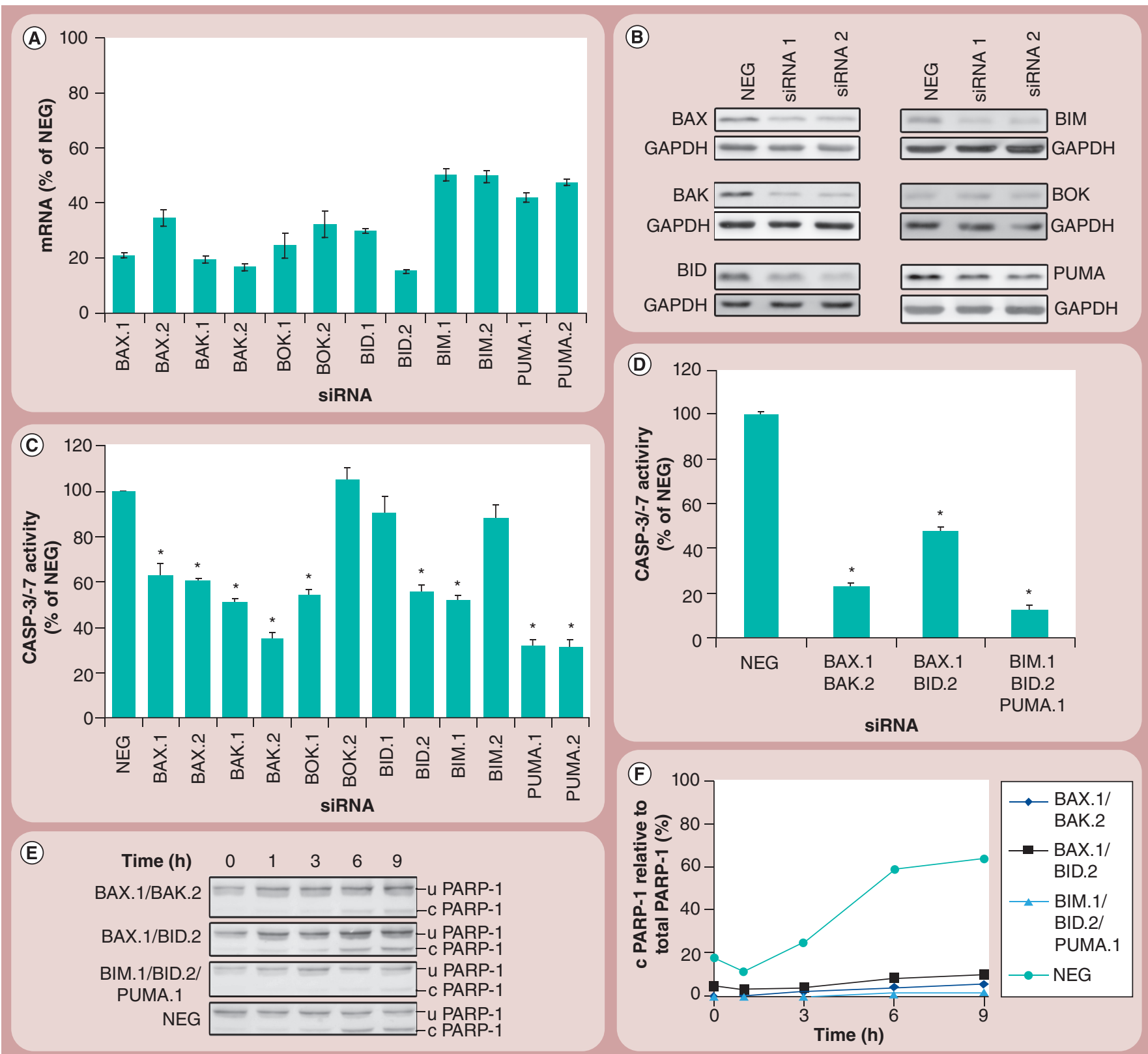

Figure 3. The mechanism of apoptosis is regulated by members of the Bcl-2 family of proteins. (A) Validation of the efficiency of the siRNA knockdown at the mRNA level by real-time quantitative PCR as described in the 'Materials \& methods' section. Bars indicate relative mRNA levels after $48 \mathrm{~h}$ in cells treated with various siRNAs, compared with cells treated with NEG siRNAs. Results shown represent the mean and standard deviations of four technical replicates and are representative of three independent experiments. All data points were significantly different $(p<0.001)$ from cells treated with NEG siRNAs. (B) Western blotting shows the efficiency of the siRNA knockdown at the protein level $48 \mathrm{~h}$ post-transfection. Results are representative of three independent experiments. GAPDH is used as a loading control. (C) CASP-3 and -7 activity of 1321N1 cells treated for $48 \mathrm{~h}$ with different siRNAs followed by $24 \mathrm{~h}$ of incubation with amine-modified polystyrene nanoparticles is shown as percentage of the activity of cells treated with NEG siRNAs. Results represent the mean and standard error of the mean from three independent experiments each with three replicates. (D) CASP-3 and -7 activity in cells transfected for $48 \mathrm{~h}$ with siRNAs targeting mRNA coding for proteins involved in BAX and BAK activation, followed by $24 \mathrm{~h}$ of incubation with amine-modified polystyrene nanoparticles is shown as a percentage of the activity of cells treated with NEG siRNAs. (E) 1321N1 cells were treated as for (D) and $30 \mu \mathrm{g}$ of protein was then subjected to western blotting to detect PARP-1 cleavage. (F) Quantification of the percentage of cleaved PARP-1 over the total PARP-1 protein was carried out by densitometry. Results represent the mean and standard error of the mean from three independent experiments, each with three replicates.

${ }^{*} \mathrm{p}<0.001$.

C PARP-1: Cleaved PARP-1; u PARP-1: Uncleaved PARP-1. 
To further validate this striking cumulative effect of the siRNAs on CASP-3 and -7 activation in preventing nanoparticle-induced apoptosis, we employed an alternative assay to determine apoptotic activity. One consequence of activation of CASP- 3 and -7 is the cleavage of PARP-1, a nuclear DNA repair enzyme that is cleaved upon induction of apoptosis [23]. Western blotting analysis and quantification of PARP-1 cleavage in cells treated with NEG siRNAs revealed a gradual accumulation of cleaved PARP-1, in response to time-dependent exposure to nanoparticles (Figure $2 F \& G$ ). The cleaved PARP-1 product was first detectable at $3 \mathrm{~h}$ after addition of the nanoparticles. A similar profile of PARP-1 cleavage was seen in cells treated with siRNAs targeting CASP-8 and -10 . By contrast, combined downregulation of CASP-9 and APAF1 or CASP-3 and -7 led to an almost complete absence of PARP-1 cleavage (Figure 2F \& G).

\section{Bcl-2 family members act as regulators of the} apoptotic pathway

In order to extend our understanding of the molecular pathways of apoptosis that were being elicited by the $\mathrm{NH}_{2}$-PS nanoparticles, the involvement of members of the Bcl-2 family of proteins was next assessed. The knockdown efficiencies were good for all the Bcl-2 homologs (BAX, BAK, BOK and BID), with at least one of the siRNAs directed against each target causing a reduction in the levels of cognate mRNA by $80 \%$ or more (Figure $3 \mathrm{~A}$ ). These results also correlated well with the protein levels detected in the cells after transfection (Figure 3B). For the two 'BH3-only' proteins studied (BIM and PUMA), the efficiency of knockdown was comparatively not as high, with only a
$50-60 \%$ decrease in the expression levels of these genes (Figure 3A). In cells treated with this panel of siRNAs, we again assessed their relative importance to the apoptotic response by measuring CASP- 3 and -7 activation. At least one of the siRNAs targeting each of these genes under test resulted in a reduction of CASP activation (Figure 3C).

Double or triple knockdowns of BAX and BAK, and their proposed activators, were performed to determine whether apoptosis could be prevented. When BAX and BAK were downregulated simultaneously, a significant reduction in CASP-3 and -7 activities (Figure 3D), together with the cleavage of PARP-1 to approximately $20 \%$ of that observed in the NEG-treated cells (Figure 3E \& F), was observed. To further understand the regulation of $\mathrm{BAX}$ and $\mathrm{BAK}$ in this system, knockdowns of BAX and BID, and of BIM, BID and PUMA, were performed. These experiments also revealed that CASP-3 and - 7 activities and PARP-1 cleavage were all significantly reduced compared with NEG-treated cells (Figure 3D-F); however, the most striking effect was obtained when BIM, BID and PUMA were silenced together. In fact, the activation of CASP-3 and -7 was reduced to levels similar to that seen in the untreated NEG cells (not shown), and the PARP-1 cleavage profile was similar to that seen in the BAX and BAK double knockdown cells (Figure 3E \& F).

To further strengthen our data, we next measured the transcriptional activation of PUMA by $\mathrm{qPCR}$ in response to nanoparticle incubation with cells. These experiments revealed a sharp and dramatic rise in PUMA expression levels between 3 and $6 \mathrm{~h}$ after nanoparticle treatment in $1321 \mathrm{~N} 1$ cells (Figure 4A). Expression of NOXA, another proapoptotic protein of

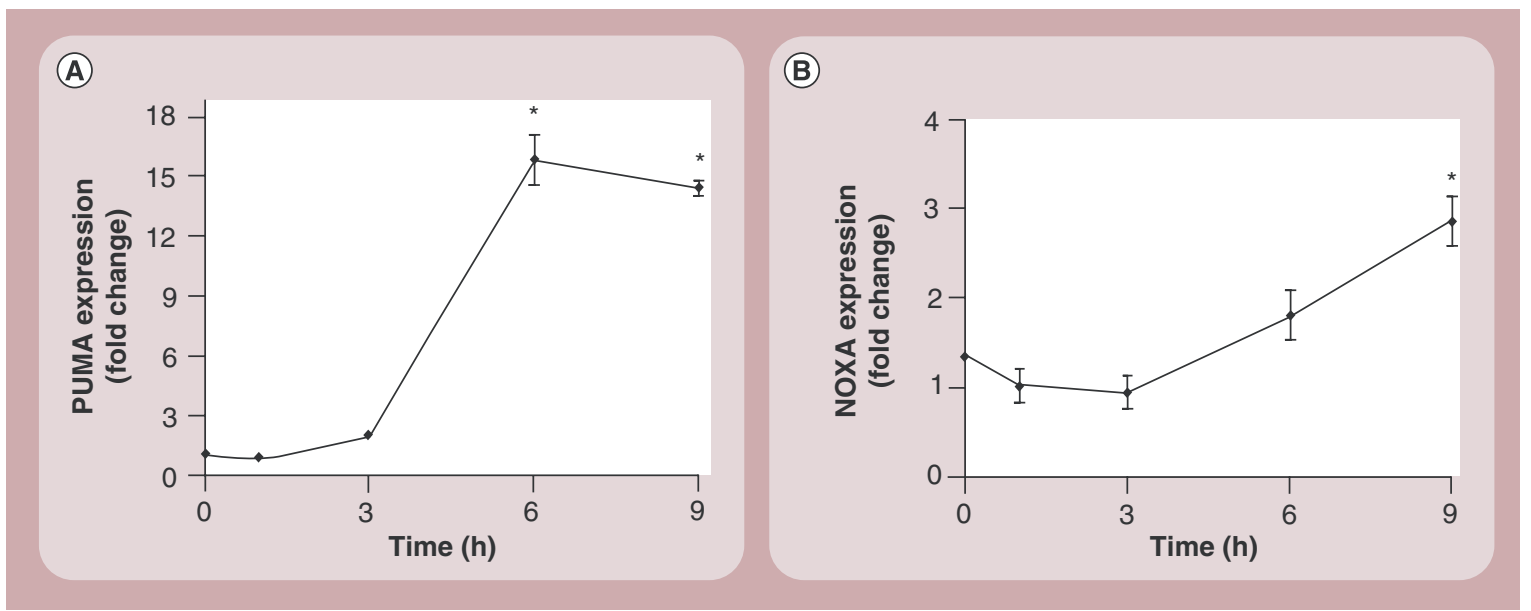

Figure 4. Amine-modified polystyrene nanoparticles induce expression of proapoptotic members of the $\mathrm{Bcl}-2$ family of proteins. Expression of (A) PUMA and (B) NOXA after exposure to amine-modified polystyrene nanoparticles for increasing lengths of time as determined by real-time quantitative PCR. Normalization was made to time-specific untreated controls. Results are representative of three independent experiments and show the mean and standard deviations from four technical replicates.

${ }^{*} p<0.001$. 
the Bcl-2 family of proteins, also increased after $6 \mathrm{~h}$ of incubation with nanoparticles (Figure 4B), but the effect was not as dramatic as that seen for PUMA.

\section{siRNA treatment is not sufficient to rescue cells} from death

Having confirmed that apoptosis was effectively blocked (as shown by the lack of CASP-3 and -7 activity, and the lack of cleavage of PARP-1) using this RNAi approach, we sought to determine whether this was, in turn, sufficient to protect the cells from nanoparticle-induced death. To this end, we measured the ATP content of the cells as an indication of their viability. Surprisingly, we observed that following treatment with the $\mathrm{NH}_{2}$-PS nanoparticles, cells depleted of BAX and BAK, BAX and BID, or BIM, BID and PUMA, all showed a similar loss of ATP levels comparable to NEG control cells, indicating that they were not protected from nanoparticle-induced death (Figure 5A). Similar results were obtained with the siRNAs targeting the different apoptotic pathways (Supplementary Figure 3). As BAX and BAK are involved in the promotion of mitochondrial permeabilization and fragmentation, we analyzed the morphology of the mitochondria in the presence of $\mathrm{NH}_{2}-\mathrm{PS}$ nanoparticles. Analysis of mitochondrial morphology has been used previously in the context of studying CASP and apoptosis regulation, and provides a powerful readout of mitochondrial health at the single cell level [24]. Using the mitochondrial chaperone HSP60 as a marker for this organelle, immunofluorescence and confocal microscopy experiments revealed that, in cells not exposed to $\mathrm{NH}_{2}$-PS nanoparticles, the mitochondria displayed their usual extended tubular morphology; however, in the presence of nanoparticles, the mitochondria were more vesicular in profile and showed reduced tubular interconnections (Figure 5B). Similar mitochondrial profiles were also seen using antibodies against manganese superoxide dismutase (data not shown). Surprisingly, this marked vesiculation of the mitochondria was not prevented by the depletion of BAX and BAK (Figure $5 \mathrm{~B}$ ). To corroborate our visual observations, we applied high-content screening analysis to the images. This revealed that the addition of nanoparticles to the cells caused only a mild reduction in the total cell cytoplasm area; however, there was a significant increase in the number of distinct mitochondrial structures, which is consistent with their vesiculation (Figure 5C). Analysis of the morphology of the mitochondria also confirmed our visual observations, with the mitochondria in nanoparticle-treated cells taking on a rounder shape (Figure 5C). The depletion of BAX and BAK was unable to rescue this phenotype.

\section{Discussion}

With the increasing use of nanoparticles for therapeutic applications in humans, the need to fully understand how they interact with living cells is becoming ever more important. One technique increasingly used to map the pathways of how cells react to particular stimuli is RNAi; however, this has never been systematically applied to study the interactions between nanoparticles and living cells. Here, we optimize and apply this technology to elucidate the molecular mechanisms of apoptosis induced by $\mathrm{NH}_{2}$-PS nanoparticles in an astrocytoma cell line. In this work, we used polystyrene nanoparticles with an amine surface modification, which confers a positive surface charge, as a model for cationic nanoparticles [9]. The mechanism by which these nanoparticles are internalized into cells remains to be established; however, it has recently been shown that on addition to the cellular medium they become coated with proteins, the so-called 'corona', that appear to protect the cells from the nanoparticles' positive charge until they accumulate in internal acidic organelles [25]. Once inside the cells, the nanoparticles then seem to be able to induce a cell death response, and our previous work indicates that apoptosis is involved [9]. Therefore, the first molecular targets we selected were the initiators of both the extrinsic (CASP-8 and -10) and intrinsic (CASP-9 and APAF1) pathways of apoptosis, and as a common downstream link between the two pathways, CASP-3 and -7 [26]. Using two independent siRNA sequences against each target, we first established the efficiency by which each siRNA downregulated the expression of its target gene. Two different approaches were used; qPCR and western blotting, to analyze the amount of mRNA and protein remaining in the cell after treatment with siRNAs, respectively. Together, these experiments revealed that for this particular group of targets, with the exception of CASP-10, the siRNA panel available was generally highly effective in terms of its ability to downregulate the target genes, and that both qPCR and western blot analysis were reliable and comparable methods to quantitatively assess the degree of downregulation.

Analysis of the phenotype of $1321 \mathrm{~N} 1$ cells transfected with siRNAs and treated with $\mathrm{NH}_{2}$-PS nanoparticles was carried out using a luminescence-based assay as described previously [9]. Initial experiments using a NEG siRNA revealed that the downregulation procedure had no effect on the sensitivity of the assay when compared with nontransfected cells. Since the addition of NEG siRNAs takes account of the transfection process, we considered this to be a more robust control and, therefore, all normalizations were carried out relative to cells treated with these siRNAs. This assay was then repeated for each of the siRNAs targeting components 


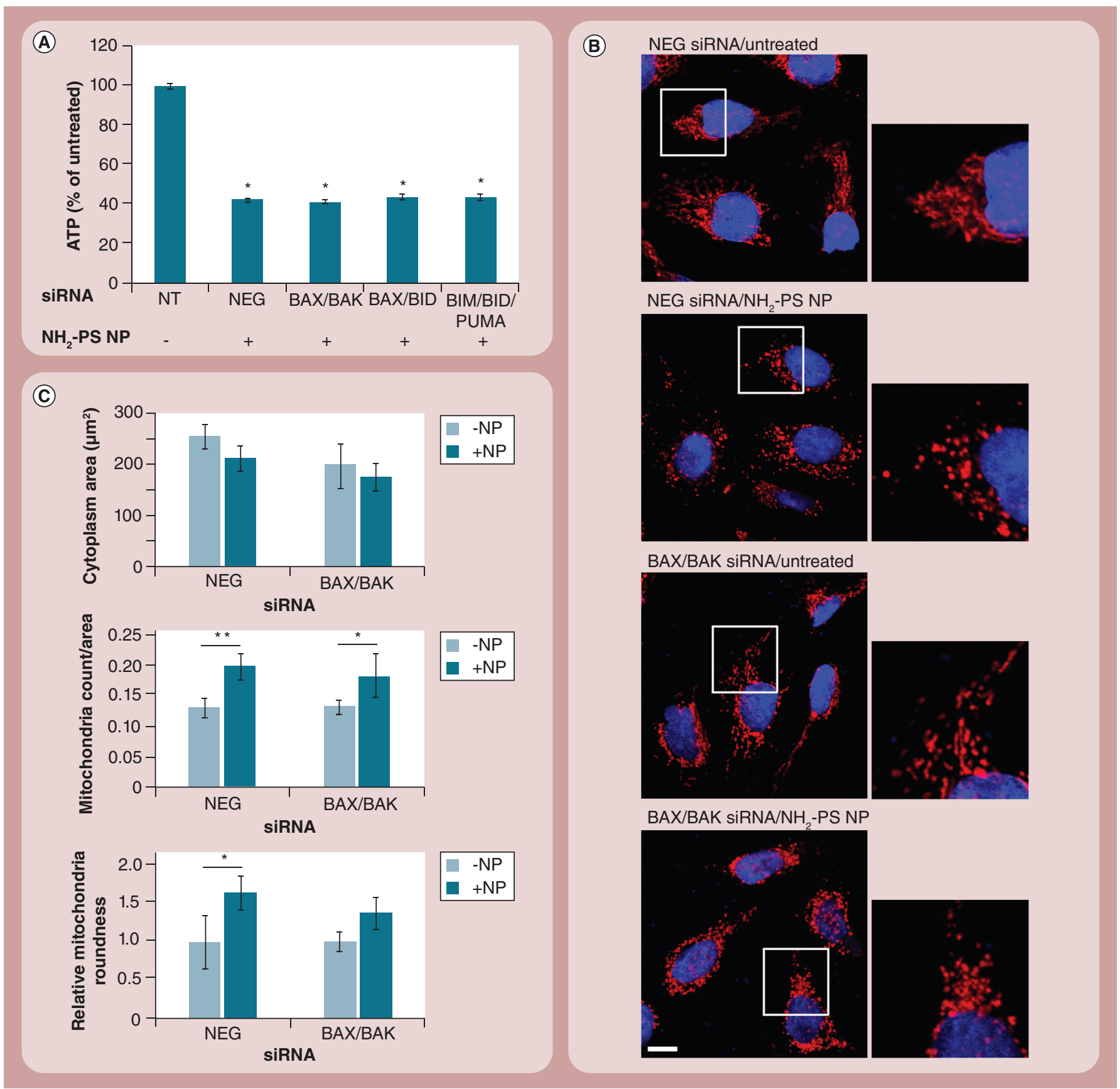

Figure 5. Amine-modified polystyrene nanoparticles induce cell death and mitochondrial fragmentation independent of BAX and BAK. (A) 1321N1 cells were transfected for $48 \mathrm{~h}$ with various combinations of siRNAs as indicated and were then either left untreated (-) or treated with $50 \mathrm{\mu g} / \mathrm{ml}$ of $\mathrm{NH}_{2}$-PS nanoparticles for $24 \mathrm{~h}(+)$. ATP was measured as an indicator of cellular viability and normalization was carried out using the nontransfected and non-nanoparticle treated sample. Results are shown as the mean and standard error of the mean and are representative of three independent experiments, each with three replicates. (B) Cells transfected for $48 \mathrm{~h}$ with various combinations of siRNAs as indicated were either left untreated or treated with $50 \mu \mathrm{g} / \mathrm{ml}$ of $\mathrm{NH}_{2}$-PS nanoparticles for 6 h, followed by immunostaining for HSP60 (red) and nuclei (blue) and confocal microscopy analysis. Mitochondrial fragmentation could not be reverted in the absence of BAX and BAK. Scale bar: $10 \mu \mathrm{m}$. (C) High content analysis of cells in (B) revealed a small decrease in cytoplasm area, and a large increase in mitochondrial count and roundness on exposure to nanoparticles. These effects could not be reverted by the depletion of BAX and BAK. Results are shown as mean and standard deviations of the mean and are representative of cells from four independent fields of view.

(A) * $p<0.001$; and (C) * $p<0.05$ and ** $p<0.01$.

$\mathrm{NH}_{2}-\mathrm{PS}$ : Amine-modified polystyrene; NP: Nanoparticle; NT: Nontransfected. 
of the two apoptotic pathways. This was performed using the siRNAs individually as well as pairing them according to the respective apoptotic pathway. The results obtained imply that the apoptosome formed by CASP-9, APAF1 and cytochrome $\mathrm{C}$ is central to the pathway of apoptosis induced by $\mathrm{NH}_{2}$-PS nanoparticles [27]. Together with our previous results [9], these experiments not only strongly implicate the intrinsic apoptosis pathway as a driver of nanoparticle-induced cell death, but also highlight the power of RNAi as a methodology to further explore the molecular basis of nanoparticle interactions with cells.

In order to increase our knowledge of the cellular machinery involved in the signaling of apoptosis upon interaction of $\mathrm{NH}_{2}$-PS nanoparticles with cells, we next studied the involvement of the Bcl-2 family of proteins. This family of proteins contains both anti- and pro-apoptotic members that modulate the apoptotic response [28]. Since not all members of this family are functionally well characterized, we decided to focus our studies on the better understood proapoptotic proteins, namely the four Bcl-2 homologs (BAX, BAK, BOK and BID) and two 'BH3-only' proteins (BIM and PUMA). Using a similar strategy to that described above, we investigated the effects of siRNAs targeting these Bcl-2 family members in $1321 \mathrm{~N} 1$ cells treated with $\mathrm{NH}_{2}$-PS nanoparticles. One interesting observation was that the level of mRNA depletion in the cells for each of the targets did not necessarily correlate with the phenotypic effects observed. The siRNAs targeting BIM and PUMA, which had displayed only modest decreases in mRNA levels, resulted in relatively strong phenotypes in terms of inhibition of CASP-3 and -7 activities (Figure 3C). By contrast, the $\mathrm{Bcl}-2$ homologs that were apparently depleted to a higher degree did not present such a striking effect. This suggests that for some of these family members at least, they may exist in the cytoplasm at carefully regulated levels, such that even minor changes in their abundance can result in dramatic phenotypic effects.

BAX and BAK, two of the proapoptotic Bcl-2 homologs studied here, are responsible for the formation of pores in the outer mitochondrial membrane (OMM) upon activation. This, in turn, can cause the release of apoptogenic molecules into the cytoplasm, such as cytochrome $\mathrm{C}$ and SMAC/DIABLO [26,28]. Inactive BAX is normally found in the cytosol, but upon activation is recruited to the OMM where it oligomerizes with BAK, with consequential formation of pores [29]. The mechanism of activation of BAX and BAK is still under debate; however, two models have been proposed. The first model postulates the direct activation of BAX and BAK by 'BH3-only' proteins, such as tBID, whereas an alternative model proposes that BAX and BAK acti- vation occurs by default through inactivation of all the antiapoptotic members of the Bcl-2 family [28,30]. Recently, another study has indicated that it is the concerted action of BIM, BID and PUMA that is necessary for activation of the BAX-and BAK-dependent cell death program [31]. PUMA itself is a potent proapoptotic regulator, that is transcriptionally activated by $\mathrm{p} 53$ or $\mathrm{CHOP}$ in response to different death-inducing stimuli [32]. PUMA is thought to work either by direct [33] or indirect [34] activation of BAX, thereby promoting the permeabilization of the OMM and apoptosome-dependent apoptosis. Our RNAi data and our observation that PUMA can be upregulated on exposure of cells to $\mathrm{NH}_{2}$-PS nanoparticles, suggest that, together with BIM and BID, PUMA is involved in regulating the activation of BAX and BAK, thereby modulating the apoptotic pathway induced by $\mathrm{NH}_{2}$-PS nanoparticles.

Examination of the literature revealed that under certain circumstances cells have the ability to change from one cell death mechanism to another [35]. Indeed, it has been reported that if a particular event inhibits apoptosis and that this occurs upstream of mitochondrial membrane permeabilization, cell death can be prevented. However, if this occurs downstream of this event, the cell will simply die by a different mechanism [36,37]. Mitochondrial fragmentation occurs early during apoptosis, preceding cytochrome $\mathrm{C}$ release, and is at least, in part, modulated by BAX and BAK via interaction with mitochondrial fission and fusion machinery [38]. In cells treated with nanoparticles, we observed a profound change in mitochondrial morphology, reminiscent of mitochondrial fragmentation, and which would be a phenotype consistent with onset of apoptosis. The fact that this fragmentation was not prevented in the double knockdown cell, might be indicative that the small amounts of BAX and BAK proteins remaining in the cells after knockdown are still sufficient to interact with the mitochondrial fission and fusion machinery and induce mitochondrial fragmentation. However, an alternative interpretation is that the cell death cascades induced by the internalization of $\mathrm{NH}_{2}$-PS nanoparticles are so strong that the cell is committed to dying by whatever mechanism is available. Indeed, others have shown in cells lacking BAX and BAK activity a necrotic form of death can be induced when normal apoptosis pathways are restricted [39].

Although in this work we analyze only one type of nanoparticle at a single concentration, these conditions are consistent with our own previous work [9] and that of others [25]. It is possible that at this particular nanoparticle concentration we do not identify all the signaling pathways that may be invoked by cell-nanoparticle interactions and, therefore, future studies will clearly need to address this possibility. In 
addition, we would also speculate that nanoparticles may activate cellular pathways beyond those associated with cell death and, therefore, further work will be needed to elucidate the molecular mechanisms underlying such crosstalk. Our pilot RNAi study undertaken here is expected to be one appropriate way to achieve this goal.

\section{Conclusion}

We have described an experimental system utilizing RNAi to study the interactions of $\mathrm{NH}_{2}$-PS nanoparticles with $1321 \mathrm{~N} 1$ cells. Moreover, this work has extended our previous results [9], showing that the formation of the apoptosome by CASP-9 and APAF1 is a central response to the presence of $\mathrm{NH}_{2}$-PS nanoparticles (Figure 6) [9,25]. This activation seems to be modulated by BAX and BAK, which are in turn regulated by BIM, BID and PUMA. However, depletion of these proteins was unable to rescue the cells from dying, suggesting that multiple signaling pathways are activated in the cell upon exposure to nanoparticles. This focused RNAi approach that we have developed here should pave the way for a more detailed under- standing of the complete range of pathways and cellular responses induced by nanoparticles.

\section{Future perspective}

The present study used $\mathrm{NH}_{2}$-PS nanoparticles as a model for cationic nanoparticles in order to understand how these nanoparticles interact with cells and induce cytotoxicity. Great expectations lie in the use of cationic nanoparticles as drug and gene delivery vectors, and so it is of the utmost importance to understand how they interact with cells at all levels. Ultimately, a greater understanding of how the physicochemical properties of nanoparticles, such as charge, affect their interaction with cells will be essential to inform their design and synthesis specific for each desired application. Appropriate design features might potentiate the effect of the drug that the nanoparticle is carrying, thereby allowing reduction of the drug dosage. The results shown here represent only the beginning of a series of studies that need to be completed to fully understand how nanoparticles interact with cells. In this work we have demonstrated that RNAi is a valuable tool for the field and we

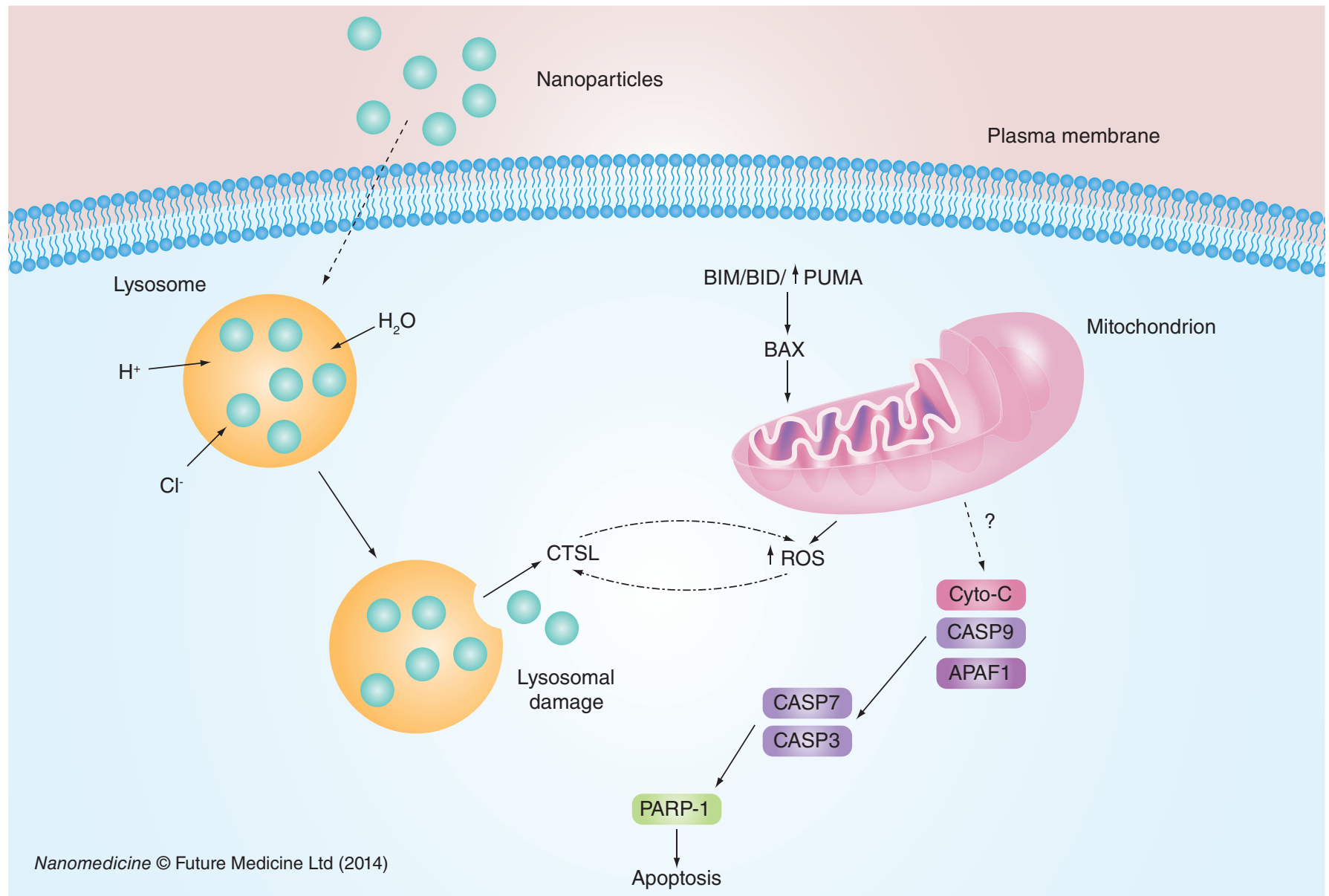

Figure 6. Cellular and molecular mechanisms of apoptosis induced by amine-modified polystyrene nanoparticles in $1321 \mathrm{~N} 1$ cells. ?: Unknown mechanism; Cyto-C: Cytochrome C; ROS: Reactive oxygen species. 
believe that, in the future, more studies will employ systematic gene knockdown to understand and modulate the effect of nanoparticles in cells. In particular, RNAi has the ability to provide a systems-level view of cellular events, and it is envisaged that large-scale studies will soon shed light on how processes such as cell signaling, migration and membrane traffic can be influenced by nanoparticles.

\section{Acknowledgements}

The authors wish to thank the UCD CSTAR team for help with statistical analysis, J Varela for flow cytometry data, and members of the JC Simpson laboratory for useful discussions.

Financial \& competing interests disclosure MG Bexiga was supported by the Portuguese Fundação para a Ciência e Tecnologia (SFRH/BD/15892/2005). The JC Simpson laboratory is supported by a Principal Investigator award (09/IN.1/B2604) from Science Foundation Ireland and by a strategic initiative award from the UCD College of Science. This work was further supported by Science Foundation Ire- land under grant number SFI/SRC/B1155 to KA Dawson. The authors have no other relevant affiliations or financial involvement with any organization or entity with a financial interest in or financial conflict with the subject matter or materials discussed in the manuscript apart from those disclosed.

No writing assistance was utilized in the production of this manuscript.

\section{Ethical conduct of research}

The authors state that they have obtained appropriate institutional review board approval or have followed the principles outlined in the Declaration of Helsinki for all human or animal experimental investigations. In addition, for investigations involving human subjects, informed consent has been obtained from the participants involved.

\section{Open access}

This work is licensed under the Creative Commons Attribution-NonCommercial 3.0 Unported License. To view a copy of this license, visit http://creativecommons.org/licenses/bync-nd/3.0.

\section{Executive summary}

\section{Establishment of RNAi as a method to study cationic nanoparticle-induced apoptosis}

- RNAi was demonstrated to effectively downregulate the expression of the selected genes.

- siRNA transfection did not interfere with the assays used to analyze apoptosis.

The apoptosome is central to the apoptotic mechanism

- CASP9 and APAF1 were shown to be necessary to the apoptotic mechanism that occurs after addition of amine-modified polystyrene $\left(\mathrm{NH}_{2}-\mathrm{PS}\right)$ nanoparticles to the cells, thereby suggesting that the apoptosome is required for the signaling process.

Bcl-2 family members act as regulators of the apoptotic pathway

- BAX and BAK, two strong proapoptotic proteins were shown to be necessary for caspase activation, suggesting that they are modulators of apoptosis.

- Downregulation of BIM, BID and PUMA, proposed modulators of BAX and BAK activity, led to a decrease in apoptotic markers to levels similar to those of untreated cells.

siRNA treatment is not sufficient to rescue cells from death

- Although a role for apoptosis in the cytotoxicity of $\mathrm{NH}_{2}-\mathrm{PS}$ nanoparticles has been established, the RNAi strategy is unable to rescue cells from death, suggesting that other mechanisms can be involved in this process.

- BAX and BAK downregulation was unable to prevent mitochondrial fragmentation.

Conclusion

- Despite being able to prevent apoptosis in 1321N1 cells, RNAi was unable to rescue cell death in cells treated with $\mathrm{NH}_{2}$-PS nanoparticles. More studies of alternative cell death pathways need to be carried out.

- The executers and the role of mitochondrial fragmentation are not yet fully understood.

- RNAi was effectively used to modulate and study the molecular mechanisms of cell death induced by $\mathrm{NH}_{2}-\mathrm{PS}$ nanoparticles. This tool could be applied to study the interactions of other types of nanoparticles with living cells.

\section{References}

Papers of special note have been highlighted as:

- of interest; $\bullet$ of considerable interest

1 Patel S, Bhirde AA, Rusling JF, Chen X, Gutkind JS, Patel V. Nano delivers big: designing molecular missiles for cancer therapeutics. Pharmaceutics 3(1), 34-52 (2011).
2 Shmueli RB, Ohnaka M, Miki A et al. Long-term suppression of ocular neovascularization by intraocular injection of biodegradable polymeric particles containing a serpin-derived peptide. Biomaterials 34(30), 7544-7551 (2013).

3 Wang Z, Niu G, Chen X. Polymeric materials for theranostic applications. Pharm. Res. doi:10.1007/s11095013-1103-1107 (2013) (Epub ahead of print). 
4 Meyers JD, Doane T, Burda C, Basilion JP. Nanoparticles for imaging and treating brain cancer. Nanomedicine (Lond.) 8(1), 123-143 (2013).

5 Oberdörster G, Stone V, Donaldson K. Toxicology of nanoparticles: a historical perspective. Nanotoxicology 1(1), 2-25 (2007).

6 Aramaki Y, Takano S, Tsuchiya S. Cationic liposomes induce macrophage apoptosis through mitochondrial pathway. Arch. Biochem. Biophys. 392(2), 245-250 (2001).

7 Iwaoka S, Nakamura T, Takano S, Tsuchiya S, Aramaki Y. Cationic liposomes induce apoptosis through p38 MAP kinase-caspase-8-Bid pathway in macrophage-like RAW264.7 cells. J. Leukoc. Biol. 79(1), 184-191 (2006).

8 Lv H, Zhang S, Wang B, Cui S, Yan J. Toxicity of cationic lipids and cationic polymers in gene delivery. J. Control. Release 114(1), 100-109 (2006).

9 Bexiga MG, Varela JA, Wang F et al. Cationic nanoparticles induce caspase 3-, 7- and 9-mediated cytotoxicity in a human astrocytoma cell line. Nanotoxicology 5(4), 557-567 (2011).

-. Demonstrates how nanoparticle-induced cell death is dependent on the charge of the nanoparticle.

10 Xia T, Kovochich M, Liong M, Zink JI, Nel AE. Cationic polystyrene nanosphere toxicity depends on cell-specific endocytic and mitochondrial injury pathways. ACS Nano 2(1), 85-96 (2008).

11 Mcstay GP, Salvesen GS, Green DR. Overlapping cleavage motif selectivity of caspases: implications for analysis of apoptotic pathways. Cell Death Differ. 15(2), 322-331 (2008).

- Demonstrates that the existing caspase substrates and inhibitors are not specific, thereby posing questions about their suitability for mechanistic studies.

12 Rual JF, Hirozane-Kishikawa T, Hao T et al. Human ORFeome version 1.1: a platform for reverse proteomics. Genome Res. 14(10B), 2128-2135 (2004).

13 Fire A, Xu S, Montgomery MK, Kostas SA, Driver SE, Mello CC. Potent and specific genetic interference by doublestranded RNA in Caenorhabditis elegans. Nature 391(6669), 806-811 (1998).

14 Rana TM. Illuminating the silence: understanding the structure and function of small RNAs. Nat. Rev. Mol. Cell Biol. 8(1), 23-36 (2007).

15 Kim DH, Rossi JJ. Strategies for silencing human disease using RNA interference. Nat. Rev. Genet. 8(3), 173-184 (2007).

16 Neumann B, Walter T, Heriche JK et al. Phenotypic profiling of the human genome by time-lapse microscopy reveals cell division genes. Nature 464(7289), 721-727 (2010).

17 Simpson JC, Joggerst B, Laketa V et al. Genome-wide RNAi screening identifies human proteins with a regulatory function in the early secretory pathway. Nat. Cell Biol. 14(7), 764-774 (2012).

18 Aza-Blanc P, Cooper CL, Wagner K, Batalov S, Deveraux QL, Cooke MP. Identification of modulators of TRAILinduced apoptosis via RNAi-based phenotypic screening. Mol. Cell 12(3), 627-637 (2003).
19 Darlington TK, Neigh AM, Spencer MT, Nguyen OT, Oldenburg SJ. Nanoparticle characteristics affecting environmental fate and transport through soil. Environ. Toxicol. Chem. 28(6), 1191-1199 (2009).

20 Calderon-Garciduenas L, Solt AC, Henriquez-Roldan C et al. Long-term air pollution exposure is associated with neuroinflammation, an altered innate immune response, disruption of the blood-brain barrier, ultrafine particulate deposition, and accumulation of amyloid $\beta-42$ and $\alpha$-synuclein in children and young adults. Toxicol. Pathol. 36(2), 289-310 (2008).

21 Jellinger KA. Recent advances in our understanding of neurodegeneration. J. Neural. Transm. 116(9), 1111-1162 (2009).

22 Chae HJ, Kang JS, Byun JO et al. Molecular mechanism of staurosporine-induced apoptosis in osteoblasts. Pharmacol. Res. 42(4), 373-381 (2000).

23 Jagtap P, Szabo C. Poly(ADP-ribose) polymerase and the therapeutic effects of its inhibitors. Nat. Rev. Drug Discov. 4(5), 421-440 (2005).

24 Peng JY, Lin CC, Chen YJ et al. Automatic morphological subtyping reveals new roles of caspases in mitochondrial dynamics. PLoS Computat. Biol. 7(10), e1002212 (2011).

25 Wang F, Yu L, Monopoli MP et al. The biomolecular corona is retained during nanoparticle uptake and protects the cells from the damage induced by cationic nanoparticles until degraded in the lysosomes. Nanomedicine 9(8), 1159-1168 (2013).

26 Boatright KM, Salvesen GS. Mechanisms of caspase activation. Curr. Opin. Cell Biol. 15(6), 725-731 (2003).

27 Riedl SJ, Salvesen GS. The apoptosome: signalling platform of cell death. Nat. Rev. Mol. Cell Biol. 8(5), 405-413 (2007).

28 Youle RJ, Strasser A. The BCL-2 protein family: opposing activities that mediate cell death. Nat. Rev. Mol. Cell Biol. 9(1), 47-59 (2008).

29 Leber B, Lin J, Andrews DW. Embedded together: the life and death consequences of interaction of the Bcl-2 family with membranes. Apoptosis 12(5), 897-911 (2007).

30 Galonek HL, Hardwick JM. Upgrading the BCL-2 network. Nat. Cell Biol. 8(12), 1317-1319 (2006).

31 Ren D, Tu HC, Kim H et al. BID, BIM, and PUMA are essential for activation of the BAX- and BAKdependent cell death program. Science 330(6009), 1390-1393 (2010).

- First report showing that BIM, BID and PUMA act in concert to activate BAX and BAK; therefore, modulating the apoptotic response.

32 Yu J, Zhang L. PUMA, a potent killer with or without $\mathrm{p} 53$. Oncogene 27(Suppl. 1), S71-S83 (2008).

33 Gallenne T, Gautier F, Oliver L et al. Bax activation by the BH3-only protein Puma promotes cell dependence on antiapoptotic Bcl-2 family members. J. Cell Biol. 185(2), 279-290 (2009).

34 Jabbour AM, Heraud JE, Daunt CP et al. Puma indirectly activates Bax to cause apoptosis in the absence of Bid or Bim. Cell Death Differ. 16(4), 555-563 (2009). 
35 Golstein P, Kroemer G. Redundant cell death mechanisms as relics and backups. Cell Death Differ. 12(Suppl. 2), 1490-1496 (2005).

36 Chipuk JE, Green DR. Do inducers of apoptosis trigger caspase-independent cell death? Nat. Rev. Mol. Cell Biol. 6(3), 268-275 (2005).

37 Kroemer G, Martin SJ. Caspase-independent cell death. Nat. Med. 11(7), 725-730 (2005).
38 Karbowski M, Norris KL, Cleland MM, Jeong SY, Youle RJ. Role of Bax and Bak in mitochondrial morphogenesis. Nature 443(7112), 658-662 (2006).

- Describes the role of proapoptotic BAX and BAK in the regulation of mitochondrial fusion and fission.

39 Zong WX, Ditsworth D, Bauer DE, Wang ZQ, Thompson CB. Alkylating DNA damage stimulates a regulated form of necrotic cell death. Genes Dev. 18(11), 1272-1282 (2004). 